\title{
An integrative ambient agent model for unipolar depression relapse prevention
}

\author{
Azizi Ab Aziz*, Michel C.A. Klein and Jan Treur \\ Agent Systems Research Group, Department of Artificial Intelligence, Faculty of Sciences, Vrije Universiteit \\ Amsterdam, De Boelelaan 1081a, 1081 HV Amsterdam, The Netherlands \\ E-mail: \{mraaziz,michel.klein,treur\}@few.vu.nl
}

\begin{abstract}
One of the challenges for persons with a history of unipolar depression is to stay healthy throughout their lifetime. In principle, having more severe prior onset cases escalates the risk to fall into a relapse. In this article, first a domain model of the process of depression, recovery and relapse is presented, and second an integrative ambient agent model to support persons from relapse is described. Based on several personal characteristics and a representation of events (i.e., life events or daily hassles) the domain model can simulate whether a human that recovered from a depression will fall into a relapse or recurrence. A number of well-known relations between events and the course of depression are summarized from the literature and it is shown that the domain model exhibits those patterns. The domain model has been mathematically analyzed to find out which stable situations exist. Second, by incorporating this domain model into an ambient agent system, the resulting integrative ambient agent model is able to reason about the state of the human and the effect of possible actions. Several simulation experiments have been conducted to illustrate the functioning of the proposed model in different scenarios. In addition, an automated verification method using Temporal Trace Language (TTL) is used to verify that the ambient agent model satisfies a number of relevant properties. Finally, it is pointed out how this model can be used in depression therapy, supported by an ambient agent.
\end{abstract}

Keywords: Human-ambient agent, temporal dynamics, relapse in unipolar depression, and decision support systems.

\section{Introduction}

Unipolar depression is a mental disorder distinguished by a persistent low mood, and loss of awareness or contentment in usual activities. Despite the modern era of pharmaceutical and holistic intervention, one of the primary problems with unipolar depression (i.e., a depression not related to other mental disorders) is that it often reoccurs [20]. Such a subsequent depressive episode is called a relapse or recurrence. Precisely, the depressive relapse stage is defined as an "episode of major depressive disorder that occurs within six months after either response or remission (no longer meeting the depression criteria)", while recurrence is a depressive episode occurring after six months have elapsed $[5,10]$. Reviewing studies of lifetime course of depression concluded that at least 60 percent of indi- viduals who have had one depressive episode will have another, 70 percent of individuals who have had two depressive episodes will have a third, and 90 percent of individuals with three episodes will have a fourth episode [5,21]. Although the risk of relapse may decline with time, even for those who remain well for 5 years after an index episode, the rate of recurrence/relapse is 58 percent [26]. The relapse nature and high rates among individuals with a history in depression have motivated many researchers on effective maintenance therapies.

However, despite the recognized magnitude of the problem of recurrence and relapse, little attention has been focused on the symptom pattern in recurrent episodes of major depression, and the related possibilities for support in an early stage, in order to prevent further development of the depressive episode. When a mechanism to monitor the

\footnotetext{
${ }^{*}$ Corresponding author.
} 
condition of individuals who have had a previous encounter with unipolar depression would be available, this would eventually improve the quality of life for a large group of persons [1,8]. There are several approaches to provide self-health interventions for depressed individuals namely; Cognitive Behavioural Therapy (CBT) [1,26], MindfulnessBased Cognitive Behavioural Therapy (MBCT) [20], and Activity Scheduling (AC) [8]. However, these approaches focus on facilitating depressed individuals to recover from depression rather than monitoring any potential future relapses.

The aim of this article is to present the basis of an intelligent ambient agent application that complements the existing approaches by providing support for relapse prevention to support humans with depression history in the long term. This ambient agent application is expected to have capabilities to understand its environment and the individual, providing a better monitoring and assessment of the situation [32].

In the past, intelligent agent technology has become an important means for increasing analysis, decision making ability and communication. This technology has not only shaped the landscape of artificial intelligence work, but also pushes the frontiers in other field, like in medical informatics. Examples can be seen in communication and cooperation between agents to manage patient care, information agents that retrieve medical information from the Internet, and multi-agent systems that assist the doctors in the tasks of monitoring and diagnosis [3]. With the advent of wearable devices, and mobile applications, new ways are created for agents to obtain more human-awareness, by human-related information gathered from sensors and based on this enhanced human-awareness will interact and react in a more knowledgeable manner [4]. Such humanaware ambient agents will be able to contribute towards the development of personal care and human wellbeing applications by harnessing vital information from human itself [32].

To realise an intelligent agent application, it is required to integrate within the application a dynamical model of the human that describes how he or she might fall into relapse/recurrence or could stay healthy. Thus, first a dynamical model is presented of the way in which humans are developing and experiencing relapse. This domain model is a crucial basic building block, comprised of a set of equations to model the dynamics of relapse and recurrence. Second, a model of an ambient agent to support persons from relapse is described, in which the do- main model is embedded. The resulting integrative ambient agent is able to reason about the state of the human and the effect of possible actions. In case a relapse or recurrence is predicted, the agent can provide to support by providing adequate remedies in an early stage.

This article is structured as follows. After an introduction of the area of depression relapse and its monitoring, first the dynamical model for relapse and recurrence of unipolar depression is described in some detail and its behaviour analyzed by means of simulation and a mathematical analysis. Next, the integrative ambient agent model is described. It covers a number of sub-models used as building blocks. The main concepts of this model are specified, and results from simulation experiments are discussed and verified. Finally, a discussion concludes this article.

\section{Monitoring relapse and recurrence of unipolar depression}

For the individuals at high risk of relapse and recurrence, there are several factors that may lead to these both stages. The combination of these factors will lead to the sudden onset, and usually is a manifestation associated with major stress. Monitoring these factors will allow an early detection of a risk on relapse. In this section, first the underlying factors that influence relapse and recurrence are discussed. Then, an overview is given of the state-ofthe-art in sensing devices in this domain.

\subsection{Underlying factors of relapse and recurrence in unipolar depresssion}

Generally, prior to relapse there might be changes in the usual symptoms of the illness, or changes in behaviour, thoughts or feelings. These changes (or symptoms) are useful warning signs. Symptoms may develop over days to weeks, though sometimes there is fearfulness, anxiety, and lowered mood over preceding months [20]. Thus, by analyzing related factors, one will be able to determine the potential risk of individuals is having a potential onset. The earlier those symptoms can be identified, the better chance there is of stopping a relapse/recurrence or reducing the severity of it. Therefore, in this section the main factors influencing the recurrence and relapse of depression as known from the literature are described. First, the effect of repeated stressful 
events is explained. Then, the knowledge about the causes of relapse and recurrence are discussed.

\subsubsection{Repeated stressful events}

Frequent stressful events (stressors) are correlated with a positive contribution to the development of recurrence and relapse [22]. Contrary to popular belief, repeated strikes, even when they are low, can have almost the same effect as a similar single instantaneous stressful event [22,25]. This can be explained by an analogy of striking a bell. Imagine when a bell is struck once, it emits a sound that is loud at first, and then decays in intensity. However, if each subsequent strike is applied before the sound of the preceding strike has diminished: the loudness will increase each time. Applying this to the real world, a single and low stressor event may initially be so miniscule that it is considered to cause no effect. However, many repeated and small stressor events will eventually lead to a higher level of potential stress than a single major stress-producing event. Therefore, the intensity of a single stressor event faced by an individual is not the only important factor, because if negative events are persistently present, they can have a stronger effect than an initial event with a higher intensity [25].

\subsubsection{Causes of relapse and recurrence}

A key step in the development of a model to represent potential onset of relapse and recurrence is to understand how this condition may recur. Stressors from the environment are the dominant components that will lead to recurrence or relapse of depression. This primary mechanism however is regulated by two main apparent predisposing factors, which influence the process as moderators that can neutralize each other. These two components are simplified as immunity and neuroticism (vulnerabilities in the personality) [18,24]. Immunity is defined as the ability to cope with heightens stressor. This ability somehow will allow individuals to attribute negative events without over generalized it as being severe. In addition, individuals with high immunity react with an appropriate level of distress and depressive affect but do not spiral into future relapse [31]. On the contrary, neuroticism has been defined as a personality trait with tendency to experience negative emotional states [28]. Individuals who score high on neuroticism are associated with people who tend to respond more poorly to environmental stress, and are more likely to interpret ordinary situations as threatening. Neuroticism is reported to be one of the predictors for future onset of depression $[10,19]$.
These factors are induced by the observed evidences that there are personal differences and conditions that will increase or decrease the onset of recurrence or relapse in any individual [31]. Furthermore, it is also related to the diathesis-stress model, a model that initially was used to explain schizophrenia. The diathesis-stress model involves predisposed factors (vulnerability) and external influences (negative event) to determine whether a person in a risk to develop a mental disorder in future $[9,19]$. In addition, in many articles, these two components are assumed to influence not only the possibility of onset of a depression, but also affect the duration of it. On the other hand there are many factors that eventually help people to sustain their well-being. These factors are closely related to: (a) coping skills, (b) being assertive, and (c) knowing when to seek help $[10,11,26]$. The first is the ability to cope with adversities. A coping skill is a behavioural and biologically wired tool which may be used by individuals to offset stressor events without correcting or eliminating the underlying condition [19].

On the basis of many theories in depression, coping responses and strategies have been most frequently divided into problem focused coping and avoidant coping responses. Problem focused coping responses allow an individual to increase the perceived control over their problem; it is proven in many studies that they are able to reduce the risk of onset of a depression [21]. They involve attempts to do something constructive about the stressful conditions that are harming an individual, rather than to make it worsen. In contrast, avoidance coping is detrimental in nature. When feelings of discomfort appear through stressor events, people find ways of not experiencing them [11]. Such a strategy may work in a very short term, but it is mostly considered as an inadequate approach of coping. The second component is being assertive. Individuals who are assertive tend to be aware of their emotions and communicate these in clear-cut manner and are able to make and meet goals and challenges through respect and perseverance [10,21]. In many cases, people with a high assertive level are more likely to be more proactive and problem focused rather than unassertive individuals.

The last component is the ability to seek social support ("having positive interaction of helpful behaviour provided to a person in need of support"). As a multidimensional concept in nature, social support also includes many other facets that may finally determine if social support is constructed such as having the ability to create a support net- 
Table 1

Features in relapse and recurrence

\begin{tabular}{|l|l|}
\hline \multicolumn{1}{|c|}{ Factors } & \multicolumn{1}{c|}{ Evidences } \\
\hline Neurotics & $\begin{array}{l}\text { Poor respond to the environmental stress, } \\
\text { and tends to exaggerate ordinary situations } \\
\text { as threatening. }\end{array}$ \\
\hline Low in immunity & $\begin{array}{l}\text { Residual symptoms, history with depression } \\
\text { onset, family history with mood disorders. }\end{array}$ \\
\hline $\begin{array}{l}\text { Lack of social } \\
\text { support }\end{array}$ & $\begin{array}{l}\text { Disengaging from social activities and } \\
\text { interaction with others. }\end{array}$ \\
\hline $\begin{array}{l}\text { Low in assertive- } \\
\text { ness }\end{array}$ & $\begin{array}{l}\text { Lack of self-esteem and poor control over } \\
\text { anger. }\end{array}$ \\
\hline $\begin{array}{l}\text { Relying on avoid- } \\
\text { ance coping skills }\end{array}$ & $\begin{array}{l}\text { Lack in problem-focused coping skills, } \\
\text { prone to comorbidities (substance abuses). }\end{array}$ \\
\hline Stressful events & $\begin{array}{l}\text { Events that can escalate or provoke relapse/ } \\
\text { recurrence. This an be divided into three } \\
\text { categories, namely; life, chronic, and daily. }\end{array}$ \\
\hline
\end{tabular}

work [11]. There are many characteristics of individuals that influence their potential abilities of seeking support or vice versa. For example, an individual who is highly neurotic, using more avoidant coping and having a lack of self esteem may not be able to request support, and later it may disengage him/herself from potential social support [10,28]. Table 1 summarizes several important features that potentially used to understand the formation of relapse.

Using these factors as a foundation, the following relations can be identified from the literature: (1) a series of smaller stressor events can lead to the recurrence or relapse; (2) stressor events directly affect the potential onset of relapse/recurrence; (3) neuroticism aggravates the effect of stressor events on the potential onset of a depression; (4) assertiveness and immunity will diminish the potential of onset, and (5) a combination of good social support and coping skills will reduce the risk of having future relapse/recurrence.

These concepts will be used to develop a formal model to describe the dynamics of relapse in unipolar depression.

\subsection{Potential sensing devices within the domain}

The field of pervasive and wearable technologies has recently witnessed an explosion of new inexpensive and distributed at all scales throughout everyday life which utilize personal-cues and information. These devices can be used in any combination of three main system environments either in a physical world, human-centred environments, or distributed computing environments [4]. Those devices are either loosely-bound to users, or can be more tightlybound to users. To take advantage of these new findings, an ambient agent model can be developed to support people that have recovered from a depression to maintain a healthy state. This model will be provide a building block to develop a system that continuously monitors the person and takes measures when there the risk on a relapse or recurrence is too high. Such technologies complement the ambient agent capability to understand about human conditions and providing solution if necessary.

In depression, a typical cause of relapse is a condition called the stressors. These stressors may derive from life, chronic, or daily events. The culmination of these factors will become overwhelming and leave a person feeling that they have lost control of their life. Using pervasive and wearable technologies, such conditions can be observed through several ambient sensors and devices. Table 2 summarizes related wearable or personal devices that can be potentially used to provide useful information for the proposed model.

As shown in Table 2, these devices can potentially be integrated to support the real world application. These devices are non-obtrusive and technically adequate to observe selected needed features needed. A stream of continuous physiological and behavioural information can be acquired from these sensors and devices [16,33]. Furthermore, in near future, this technology will be available for personal

Table 2

Potential sensors and devices

\begin{tabular}{|l|l|l|}
\hline Factors & Signals/Activities/Information Measured & Sensory Devices/Source \\
\hline Neuroticism & Heart rate, skin conductance, self report & $\begin{array}{l}\text { Blood volume pressure (BVP), galvanic skin response (GSR) } \\
{[13,21,27,29]}\end{array}$ \\
\hline Avoidance coping & $\begin{array}{l}\text { Alcohol level in sweat, medicine intake, } \\
\text { drug intake }\end{array}$ & $\begin{array}{l}\text { Passive alcohol sensor, medicine box (MEMS), transdermal } \\
\text { alcohol sensor (TAS), sweat patch [3,17,30] }\end{array}$ \\
\hline Social support and interaction & $\begin{array}{l}\text { Interaction with friends, acquaintances, } \\
\text { colleagues }\end{array}$ & $\begin{array}{l}\text { Mobile phone, personal digital assistant (PDA), e-mail applica- } \\
\text { tion [8,14,16] }\end{array}$ \\
\hline Immunity & Prior onset, clinical history, self report & $\begin{array}{l}\text { Record from related database, web-based questionnaires (mobile } \\
\text { phone, PDA) [3,6,12] }\end{array}$ \\
\hline Assertiveness & Self report, personal information & Web-based questionnaires (mobile phone, PDA) [3,8,12] \\
\hline
\end{tabular}


usage at affordable cost [30]. A detailed discussion on these devices and signals is beyond the scope of this article.

\section{A dynamical domain model of relapse and recurrence in unipolar depression}

Based on the analysis of depression relapse as given in the previous section, it is possible to specify a domain model for this process. A domain model is used to provide a basic reference to understand the whole function of a selected domain. In general, a domain model could be used to describe physical phenomena, physiological and cognitive phenomena, or behavioural process of human interaction within environment [2]. By incorporating the domain model with an ambient agent model, the agent gets an understanding of the processes of its environment. The model is designed in a way to be tailored with several other sub-models.

In this domain model, there are four major components that will represent dynamic interactions of human agent abilities involved in recurrence/relapse namely; environment, personality, social support, and coping strategies (as briefly explained in Section 2.1.2). By combining these characteristics together, it will allow a hypothesis or expected behaviour for the human agent to be monitored [2]. The dynamics of these concepts are as follow. In general, negative events from the environment will generate life stress. Basically, there are three types of events, namely; life, chronic and daily events [10]. A life event is defined as changes in an individual's life that are likely to have an impact on subsequent behaviour. Typical negative life events are divorcement, or death of a close family member. While, a chronic event refers to the individual condition such as having a disease and a daily event is related to typical daily hassles but without control will accumulate to cause stress. Traffic congestion is one of the examples to illustrate the negative daily event.

However, the eminence effect of these negative events will be initially by two predisposing factors, where immunity will mitigate the risk of viewing those events as being severe and global. If the immunity level is higher than the perceived negative events, then it will reduce the level of neuroticism throughout time. The current neuroticism level will influence the ability to seek help, assertiveness, and coping. Normally, a high neuroticism level is associated with a low chance to receive help, a lower assertiveness and a higher avoidance coping skill.

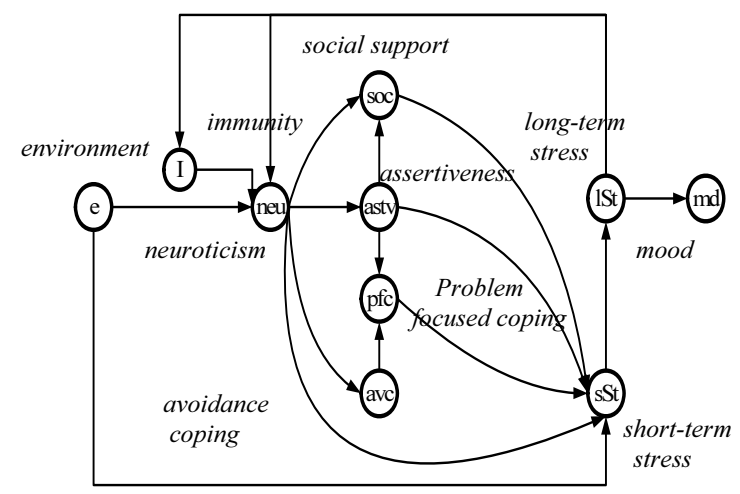

Fig. 1. Global relationships of variables involved in the domain model.

The assertiveness level has a positive effect on the social support and problem focused coping. In the model, it is assumed that every negative event will influence the formation of short term stress. A short term stress level also corresponds positively with neuroticism, but corresponds negatively with a combination of assertiveness, social support and a problem focused coping level. Throughout time, a constant high short term stress will cause a long term stress to be high, which later influences the current state of individual's mood. Prolonged exposure towards a high long term stress will escalate the current state of neuroticism and later reduce the current level of individual's immunity [15].

Once the dynamical relationships in the domain model have been determined, the model can be formalized. In the formalization, those important concepts are translated into several interconnected nodes. These nodes are designed in a way to have values ranging from 0 (low) to 1 (high). Figure 1 depicts the global interaction between these nodes. The interaction will determine the new value of it, either by a series of accumulations or an instantaneous interaction for each node. The description of these formalizations is described as the following.

\section{Stressor events}

In the model, the stressor events $(e)$ are generated by simulating potential effects throughout $t$ time using weighted sum of three types of events; life (le), chronic (ce), and daily (de) events.

$$
e(t)=w_{1} \cdot l e(t)+w_{2} \cdot c e(t)+w_{3} \cdot d e(t) .
$$

The role of these factors in the model is to represent a series of events. Stressors are seen as very intense when $e(t) \rightarrow 1$, and no stressors are represented by $e(t) \rightarrow 0$. 


\section{Dynamics of neuroticism}

In this model, the neurotic level (neu) describes the interactions between environment $(e)$, personal immunity trait $(I)$, and prior exposure to long-term stress $(l S t)$, in a time interval between $t$ and $t+\Delta t$. Here, $\alpha_{\text {neu }}$ is a parameter for a change rate, and $\beta_{\text {neu }}$ is a parameter for the contribution effect of the previous neurotic rate in this equation.

$$
\begin{aligned}
n e u(t+\Delta t)= & n e u(t)+\alpha_{\text {nеu. }} .(1-e u(t)) \cdot[(f(e(t), I(t)) \\
& . l S t(t))-\beta n e u . n e u(t))] . \Delta t
\end{aligned}
$$

where, $f(e(t), I(t))$ is a logistic unit function,

$$
f(e(t), I(t))=1 /\left(1+\eta e^{-\alpha(e(t)-I(t))}\right) .
$$

The logistic unit function is implemented to provide a smooth transition between two different values in environment and immunity.

Social support, problem focused coping, assertiveness, and immunity

Social support ( $S O c$ ) is computed by multiplying the factor of being assertive by the ability of less or non-neurotic. Problem focused coping $(p f c)$ is also computed with the same approach, but with a negative association in avoidant coping $(a v c)$. The $\alpha_{a v c}$ is proportional rate for the effect of neurotic level in $a v c$.

$$
\begin{aligned}
& \operatorname{soc}(t)=\operatorname{astv}(t) .(1-n e u(t)) \\
& p f c(t)=\operatorname{astv}(t) .(1-\operatorname{avc}(t)) \\
& \operatorname{avc}(t)=\alpha_{a v c} \cdot n e u(t)
\end{aligned}
$$

Assertiveness (astv) depends on the interaction between the normal assertive value within an individual and the condition of being less or non-neurotic. The immunity (I) level interaction also having a similar behavior, but it is related to the interaction in a long term stress level.

$$
\begin{gathered}
\operatorname{astv}(t)=\alpha_{a s t v} \cdot \operatorname{ast} v_{\text {norm }}+\left(1-\alpha_{\text {astv }}\right) . \\
(1-n e u(t)) . \operatorname{ast} v_{\text {norm }} \\
I(t)=\alpha_{I} I_{\text {norm }}+\left(1-\alpha_{I}\right) .(1-l s t(t)) . I_{\text {norm }}
\end{gathered}
$$

Dynamics of short term stress, long term stress, and mood

Short term stress ( $s S t)$ is modeled by instantaneous relationships between the environment, neurotic level, and reducer components, $\psi$ (a combination of social support, assertiveness, and problem focused coping). Long term stress (lSt) is primarily contrib- uted the accumulation exposure towards short term stress and later will influence the level of $\operatorname{mood}(m d)$ in a time interval between $t$ and $t+\Delta t$.

$$
\begin{aligned}
& s S t(t)=\beta_{s s t} \cdot e(t)+\left(1-\beta_{s s t}\right) \cdot n e u(t) .(1-e(t)) .(1-\psi)(8) \\
& l S t(t+\Delta t)= l S t(t)+\alpha_{l s t} \cdot(1-l S t(t)) \\
&\left(s S t(t)-\beta_{l s t} l S t(t)\right) . \Delta t \\
& m d(t+\Delta t)= m d(t)+\eta_{m d \cdot}(1-m d(t)) \\
&\left(l S t(t)-\beta_{m d \cdot} m d(t)\right) . \Delta t
\end{aligned}
$$

where $\eta_{m d}, \beta_{m d}, \alpha_{l S t}, \beta_{s S t}$, and $\beta_{l S t}$ denote the proportion change rates for all respective equations.

\section{Simulation results for the domain model}

In this section, it is described how the dynamical domain model for relapse and recurrence of unipolar depression was executed to simulate a number of scenarios with a variety of different conditions of individuals. To this end software to generate simulation traces was developed in Visual Basic.NET.

Three example scenarios are shown: a healthy individual $(A)$, an individual with a potential risk of relapse and recurrence $(B)$, and an individual with severe conditions $(C)$. The initial settings for the different individuals are the following (neu $(t=0)$, ast $\left._{\text {norm }}, I_{\text {norm }}\right) ; A(0.1,0.8,0.8), B(0.5,0.5,0.4)$, and $C(0.8,0.2,0.1)$. In all cases, the initial long term stress and mood value is initialized as $0.3,0.1$ respectively. Corresponding to these settings, the level of severity (or potential onset) is measured, defining that any individuals scored more than 0.5 in their mood level (within more than 336 time steps) will be considered as reaching the recurrent or relapse stage. There are several parameters can also be varied to simulate different invidual characteristics. However, these simulations used the following parameters settings: $t_{\max }=1000$ (to represent a monitoring activity up to 42 days), $\Delta t=0.3, \alpha_{l s t}=0.3, \eta_{m d}=$ $0.2, \beta_{s s t}=0.3, \alpha_{I}=0.7, \alpha_{a s t v}=0.5, \alpha_{a v c}=0.5$, and $\alpha_{\text {neu }}=0.4$. These settings were obtained from previous systematic experiments to determine the most suitable parameter values in the model.

\section{Result \# 1: Simulation trace for prolonged- fluctuating stressor events}

During this simulation, each type of individual has been exposed to an extreme stream of stressor events, with a rapid alteration between each corresponding event. This kind of pattern is comparable 


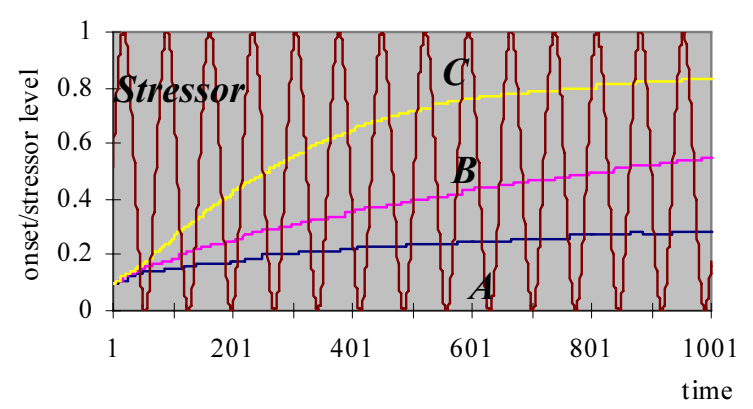

Fig. 2. Relapse/recurrent onset for each individual in prolonged stressor events.

to the repeated strike event, where stressor events always occur when the previous events were ended.

In this simulation trace, it shown that an individual $C$ (high neurotic, low in assertive and immunity) tends to get into onset much faster compared to other individuals. Note that the individual $B$ (moderate neurotic, assertive, and immunity) shows a gradual increasing level of potential onset and possibly will experience relapse/recurrent if that individual is having constant exposure towards stressors. Individual A however is less prone to develop a potential onset condition within a short period of time.

\section{Result \# 2: Simulation trace for decrease stressor events}

This simulation trace shows two types of periods, one with a very high constant and with a very low constant stressor event. These events occurred in a constant behaviour for a certain period of time (approximately within 20 days).

Also here it can be seen that individual $C$ gets into a bad mood much faster than the others. Moreover, even at the end of the simulation time, the mood of individual $C$ is worse than the mood of the other two individuals. Using a similar experimental setting, with $t_{\max }=10,000$, the end of the experimental results show all individuals will have a normal mood level.

\section{Result \# 3: Simulation trace with social support, and problem focused coping skills (reducer)}

As initially discussed in Section 2.1, a combination of social support, and problem focused coping skills is expected to help any individuals to reduce potentially risk in relapse/recurrence. The combination of these factors will be represented by $R_{A}, R_{B}$, and $\mathrm{R}_{\mathrm{C}}$ for the respective human agents. To visualize the effect of these, frequently repeating low stressor events were simulated. Figure 4 illustrates

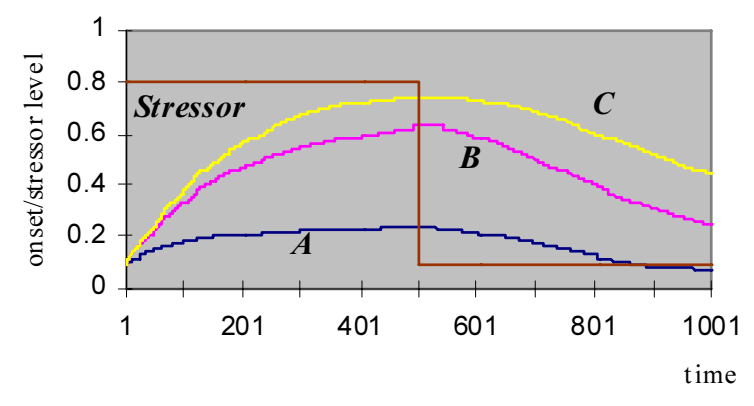

Fig. 3. Relapse/recurrent onset for each individual in fluctuated stressor events.

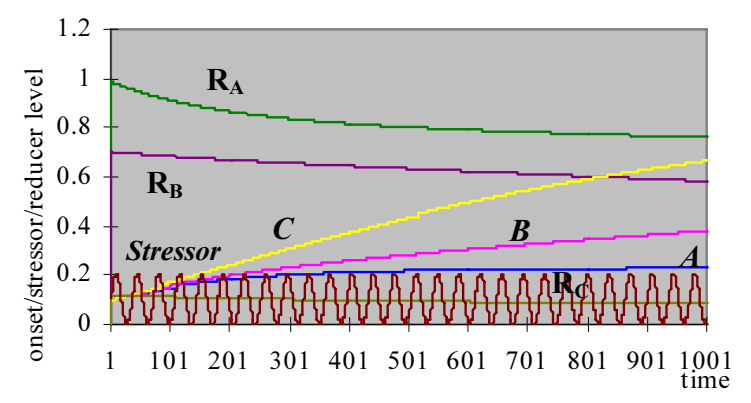

Fig. 4. Relapse/recurrent onset for each individual with a combination of reducer.

how these combinations, mood levels and stressor events are influencing each other.

Figure 4 shows that a healthy individual $(A)$ has much higher reducer factors than less healthy individuals. These reducing factors limit the effect of the incoming stressors. Also it can be seen that the reducer factors decrease over time, due to the relatively low but frequent stressors. The patterns for the different individuals are the same as in Fig. 2, although the final mood level is lower in Fig. 4 because of the less intense stressors fluctuation.

To wrap up these experimental results, the simulation traces described above satisfactorily explain the relations as summarized in Section 2. In all simulation traces, it is shown that individuals with higher assertiveness, immunity and less neurotic levels develop less often a relapse compared those who are not. In addition, a higher neurotic level eventually aggravates the potential risk of onset, as illustrated in all simulation traces. The effects of stressor events on relapse/recurrence onset are also exemplified. It is apparent that frequent or high stressor events contribute to the potential risk of onset. Furthermore, the effect of the reducers is also examined, where in Fig. 4, it depicts that when the reducer level is decreasing, the person is also prone to a relapse, or vice versa. This distillation of above 
Table 3

Equilibrium equations

\begin{tabular}{|c|c|}
\hline Variable & Equations \\
\hline neu & $n e u=1$ or $e \leq I$ and $n e u=0$ or $e>I$ and $\beta_{\text {neu }} n e u=l S t$ \\
\hline astv & $a s t v=a s t v_{\text {norm }}-\left(1-\alpha_{a s t v}\right) . n e u$. astv norm \\
\hline soc & soc $=\operatorname{astv} \cdot(1-n e u)=\left(a_{s t v_{n o r m}}-\left(1-\alpha_{a s t v}\right) . n e u . a s t v_{n o r m}\right)(1-n e u)$ \\
\hline$a v c$ & $a v c=\alpha_{a v c} . n e u$ \\
\hline$p f c$ & $p f c=\operatorname{astv} \cdot(1-a v c)=\left(a s t v_{\text {norm }}-\left(1-\alpha_{a s t v}\right)\right.$. neu. astv norm $)\left(1-\alpha_{\text {avc. }}\right.$. eu $)$ \\
\hline$I$ & $I=I_{\text {norm }}-\left(1-\alpha_{I}\right)$. lSt. $I_{\text {norm }}=I_{\text {norm }}-\left(1-\alpha_{I}\right)$. lSt. $I_{\text {norm }}$ \\
\hline$s S t$ & 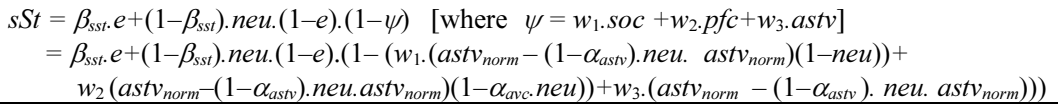 \\
\hline$l S t$ & $l S t=1 \quad$ or $s S t=\beta_{l s t} . l S t$ \\
\hline$m d$ & $m d=1$ or $m d=l S t / \beta_{m d}$ \\
\hline
\end{tabular}

evidences and traces illustrates that this model reflects the basic relations that are known to influence relapse and recurrence, given certain criteria of events and personality attributes.

\section{Mathematical analysis of the domain model}

In this section the equilibria are analyzed that may occur under certain conditions. The equilibria describe situations in which a stable situation has been reached. Those equilibria are interesting as it should be possible to explain them using the knowledge of the domain that is modeled. As such, the existence of reasonable equilibria is an indication for the correctness of the model. To analyze the equilibria, the available temporal and instantaneous equations are filled with values for the model variables such that the derivatives or differences between time point $t$ and $t+\Delta t$ are all 0 (in particular for neuroticism, long term stress and mood). Moreover, for an equilibrium, the external input is also assumed to be constant. To start, for an equilibrium for neuroticism it holds as:

$$
(1-n e u) \cdot\left(f(e, I) \cdot l S t-\beta_{\text {neu }} \cdot n e u\right)=0
$$

This is equivalent to;

$$
n e u=1 \text { or } n e u=f(e, I) . l S t / \beta_{\text {neu }},
$$

where $f(e, I)=1 /\left(1+\eta e^{-\alpha(e-I)}\right)$.

Assuming high steepness of the threshold function provides the cases $e \leq I$ (where $f(e, I)=0$ ) or $e>I$ (where $f(e, I)=1)$. Under this assumption the cases are $n e u=1$ or $n e u=0$ and $e \leq I$ or neu $=$ $l S t / \beta_{\text {neu }}$ and $e>I$. For an equilibrium for assertiveness it holds:

$$
a s t v=a s t v_{n o r m}-\left(1-\alpha_{a s t v}\right) . n e u . a s t v_{n o r m}
$$

Meanwhile, for an equilibrium for immunity it holds:

$$
\begin{aligned}
I & =\alpha_{I} \cdot I_{\text {norm }}+\left(1-\alpha_{I}\right) \cdot(1-l s t) \cdot I_{\text {norm }} \\
& =I_{\text {norm }}-\left(1-\alpha_{I}\right) \cdot \text { lSt. } I_{\text {norm }}
\end{aligned}
$$

For an equilibrium for long term stress it holds (1 $l S t) .\left(s S t-\beta_{l s t} l S t\right)=0$, which is equivalent to $l S t=1$ or $s S t=\beta_{l s t} l S t$. For an equilibrium for mood it holds $(1-m d) .\left(l S t-\beta_{m d} m d\right)=0$ which is equivalent to $m d=1$ or $m d=l S t / \beta_{m d}$. Table 3 provides a summarization of these equilibria.

From the equillibria analysis, it turns out that all values can be expressed in terms of either neu or $l S t$. In Table 3, the values astv, soc, avc, pfc have been expressed in $n e u$, and the values $m d, s S t, I$ have been expressed in $l S t$. Then by the equation for short term stress the value $l S t$ can be expressed in neu.

$$
\begin{aligned}
& \beta_{l s t} l S t=\beta_{s s t} . e+\left(1-\beta_{s s t}\right) . n e u .(1-e) .\left(1-\left(w _ { 1 } \cdot \left(a s t v_{n o r m}-\right.\right.\right. \\
& \left.\left.\left(1-\alpha_{\text {astv }}\right) . n e u \text {. ast } v_{\text {norm }}\right)(1-n e u)\right) \\
& +w_{2}\left(\text { ast }_{\text {norm }}-\left(1-\alpha_{\text {astv }}\right) \text {. neu. ast } \text { norm }_{\text {nor }}\right) \\
& \left.\left(1-\alpha_{\text {avc }} . n e u\right)\right)+w_{3} .\left(a s t v_{\text {norm }}-(1-\right. \\
& \left.\left.\left.\alpha_{\text {astv }}\right) \text {. neu. ast } v_{\text {norm }}\right)\right) \text { ) }
\end{aligned}
$$

From the equation for neuroticism two cases occur; $e \leq I$ and neu $=0$, or $e>I$, and $\beta_{\text {neu neu }}=l S t$. These cases will be addressed in some more detail.

Analysis of case $e \leq I$ and neu $=0$ :

In this case, the following values are found:

$$
\begin{aligned}
& n e u=0, \quad a s t v=a s t v_{\text {norm }}, \\
& s o c=a s t v_{\text {norm }}, \quad a v c=0, \\
& p f c=a s t v_{\text {norm }}, \\
& s S t=\beta_{\text {sst. }} e, l S t=\beta_{\text {sst. }} . e / \beta_{l s t}, I=I_{\text {norm }}-\left(1-\alpha_{I}\right) .
\end{aligned}
$$




$$
\begin{gathered}
\beta_{\text {sst. }} \text { e } / \beta_{l s t} . I_{\text {norm }}, \\
m d=1 \text { or } m d=\beta_{s s t} \text { e } / \beta_{l s t} \beta_{m d}
\end{gathered}
$$

Here the condition $e \leq I$ is equivalent to:

- $e \leq I_{\text {norm }}-\left(1-\alpha_{I}\right) . \beta_{\text {sst }}$ e $/ \beta_{\text {lst. }} I_{\text {norm }}$,

- $e\left(1+\left(1-\alpha_{I}\right) \cdot \beta_{\text {sst. }} / \beta_{\text {lst. }} I_{\text {norm }}\right) \leq I_{\text {norm }}$,

- $e \leq I_{\text {norm }} /\left(1+\left(1-\alpha_{I}\right) \cdot \beta_{\text {sst. }} / \beta_{\text {lst. }} I_{\text {norm }}\right)$

These conditions illustrate the generic condition that an extremely healthy individual (not neurotic at all) that encounters only events that are less intense than its immunity level will never develop a relapse [26].

Analysis of case $e>I$ and $\beta_{\text {neu }}$ neu $=l S t$ :

In this case the equation becomes:

$$
\begin{aligned}
& \beta_{\text {lst }} \beta_{\text {neu }} \text { neu }= \\
& \beta_{\text {sst }} . e+\left(1-\beta_{\text {sst }}\right) . n e u .(1-e) \cdot\left(1-\left(w_{1}\right. \text {. }\right. \\
& \left(\text { ast }_{\text {norm }}-\left(1-\alpha_{\text {astv }}\right) \text {. neu. ast } v_{\text {norm }}\right) \\
& (1-n e u))+w_{2}\left(a_{s t v_{n o r m}}-\left(1-\alpha_{a s t v}\right) . n e u . a_{s t v_{n o r m}}\right) \\
& \left.\left(1-\alpha_{a v c} . n e u\right)\right)+w_{3} .\left(a_{s t v_{n o r m}}-\left(1-\alpha_{a s t v}\right)\right. \text {. neu. } \\
& \text { ast } \left.\left.\left(v_{\text {norm }}\right)\right)\right)
\end{aligned}
$$

Rewriting this equation in general, provides an equation of third degree, which for given values of the parameters can be solved in an algebraic manner or numerically. For some special cases of parameter values the equation becomes simpler. For example, when $\alpha_{a s t v}=1$, it becomes a quadratic equation:

$$
\begin{aligned}
& \beta_{\text {lst }} \beta_{\text {neu }} \text { neu }= \\
& \beta_{\text {sst }} \cdot e+\left(1-\beta_{\text {sst }}\right) . n e u \cdot(1-e) \cdot\left(1-\left(w_{1} . \text { astv }_{\text {norm }} \cdot(1-n e u)\right.\right. \\
& \left.+w_{2} \operatorname{astv}_{\text {norm }}\left(1-\alpha_{\text {avc. }} \text { neu }\right)\right) \\
& \left.\left.\left.+w_{3} \text {. } \text { ast }_{\text {norm }} \text { neu. ast } v_{\text {norm }}\right)\right)\right)
\end{aligned}
$$

This situation describes how an individual that encounters events which are more intense than its immunity level will not change, if his long-term stress level is in balance with his level of neuroticism [18].

\section{The integrative ambient agent model}

The second major contribution of this article is the design of an integrative model for an ambient agent to support persons recovered from a depression. In order to achieve this, an approach has been followed in which the dynamical domain model for depression is integrated in the model that describes the functioning of the ambient agent. By integrating the domain model, the ambient agent will be able to reason about the human and environmental processes. It is important to have such capabilities, since

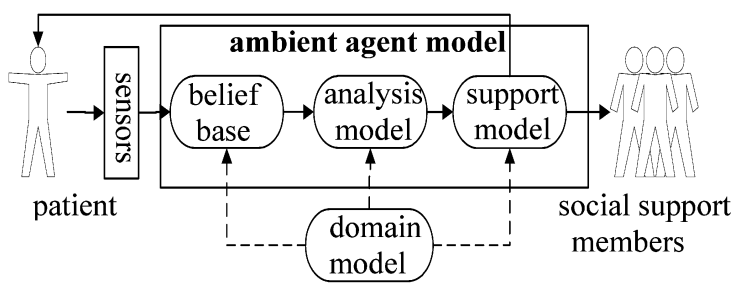

Fig. 5. Integrative ambient agent model.

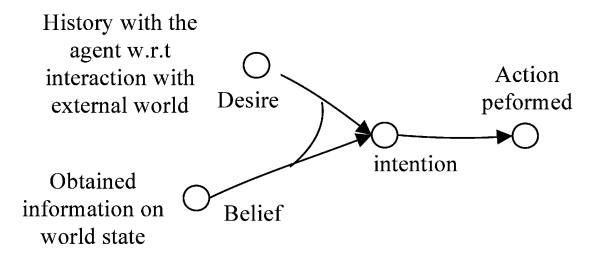

Fig. 6. The BDI structure.

an ambient agent should be aware of human behaviours and states $[4,32]$. Through this mechanism, the agent will use this knowledge to provide related actions related to the predicted state of the human and the environment.

In Fig. 5, the solid arrow indicates information exchange between processes, and the dotted arrow represents the integration process of the domain model within the ambient agent models. The following sub-sections will discuss in more detail the different elements within the integrative ambient agent model.

\subsection{Belief-Desire-Intention structure}

Basically, the Belief-Desire-Intention (BDI) structure represents the beliefs as corresponding to information the agent has about the world, while desires correspond to states of actions that the agent would wish to be executed, and intentions represent actions that the agent has committed to accomplish [23]. Figure 6 depicts the overall functioning of the BDI model.

The term belief represents that what an agent believes about the world (or another agent) may not necessarily be true all the time and may change over time. As for the integrative agent model, the BDI structure will be used as a foundation for most of the properties.

\subsection{Belief base}

The main function of the belief base is to generate initial beliefs (basic and derived beliefs) from the 


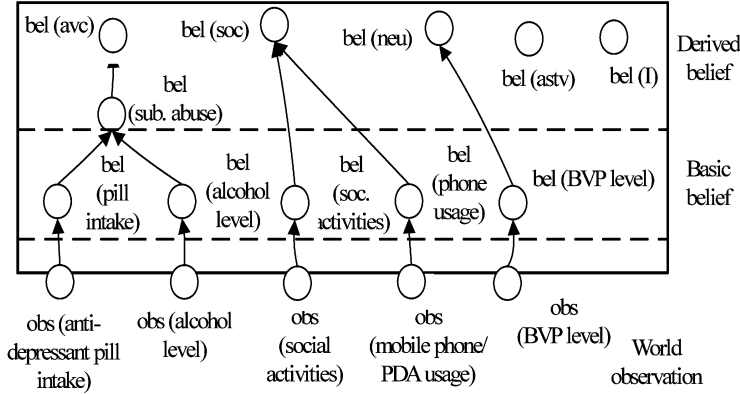

Fig. 7. Basic and derived belief in belief base.

ambient agent's observation about the person's condition (refer to Fig. 7). From this perspective, the observed beliefs and its interdependencies can be distinctively known and grouped.

Basic beliefs refer to beliefs related to the observation, while derived beliefs are based on derivations using the domain model [2]. One of the advantages to have such concept is it allows future extension of the model. For example, if there is a new method (or sensor) can be used to measure avoidant coping, it is easily can be added as a basic belief for a new observation, and append it with the existing avoidant coping belief. In addition, another model can make use this set of related beliefs without having to generate a new one.

\subsection{Analysis model}

A very important aspect to determine the risk of relapse or recurrence is the continuous evaluation of changes in selected physiological and behavioural features within the person. In the analysis model, a set of different combination of several generated beliefs is analyzed. By analysing these combinations, the person's potential risk in relapse or recurrence can be monitored and predicted. Three important steps were taken to design this model. First, the information from the domain model has been captured and abstracted. This information provides the dynamic relations in the model. The relationships can be grouped as follows $[3,21,26]$ :

(1) relations from observable features in person to beliefs about observable states,

(2) relations affecting beliefs about human conditions, such as avoidance coping, social support interaction, neurotic, assertiveness and immunity, and

(3) relations from beliefs on human condition to an assessment of his state w.r.t. relapse risk.

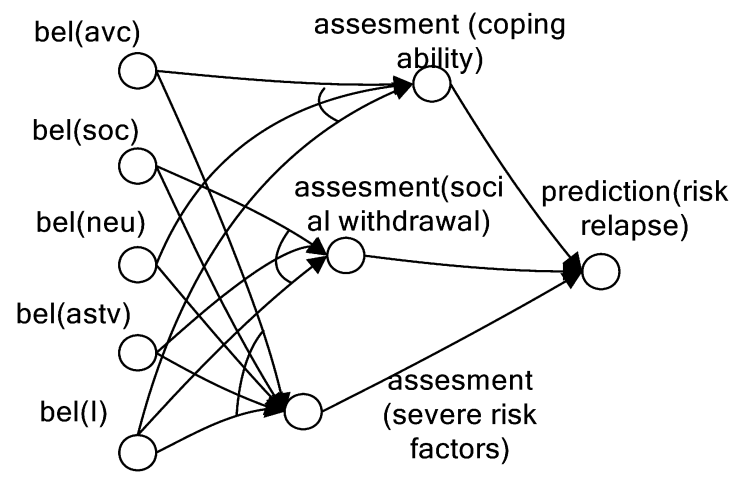

Fig. 8. Causal graph of the analysis model.

Figure 8 depicts these relationships in a causal graph.

The next step was to formalize these concepts in logical atoms. Once these concepts have been identified, the temporal relations and properties for these concepts within the ambient agent can be formally described [32]. These properties were designed to predict the risk of relapse/recurrence in the analysis model and to provide the basis for specific action selection in the support model.

\subsection{Support model}

For a person at a high risk of relapse or recurrence, necessary actions are needed to curb the onset stage. The ambient agent can use the results from analysis model to generate support actions for the person. The implementation of BDI in the support model provides an action selection process, dedicated to decide which action should be chosen. On the conceptual level, the implementation of the BDI mechanism in the support model is illustrated in Fig. 9.

This has been obtained by compiling the combination of relations in the domain model between risk factors and long term mood into direct relations between the assessment of a specific risk and specific interventions.

\subsection{Ontology and specifications}

To specify properties on dynamics relationship, the ontology of the model was designed using predicate calculus. For example, any agent ability to observe the frequency level of pill intake can be expressed as

observed(X:AGENT, pill_intake( F:FREQ_LEVEL)). 


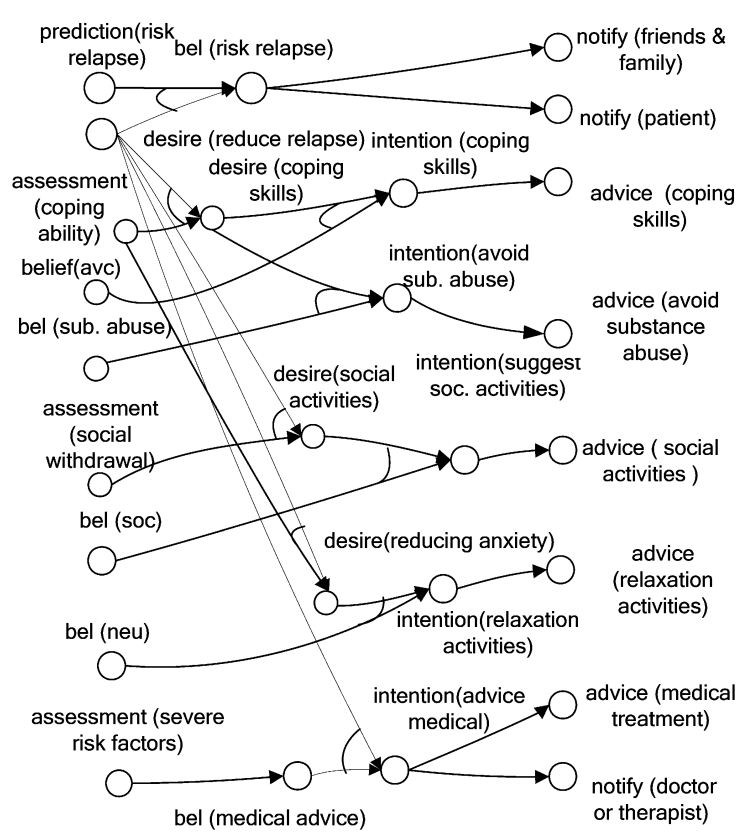

Fig. 9. Action selection process in the support model using the BDI concept.

Ontology for agent's observation: Observation using several sensors (input from person-world interaction). The agent observes human's condition through pill intake activities, alcohol compound in a blood stream, blood pressure level, phone usages, and social interaction with the social group.

observed(X:AGENT, pill_intake(F:FREQ_LEVEL))
observed(:X:AGENT, alcohol_level(L:LEVEL))
observed(X:X:AGENT, BVP_level(L:LEVEL))
observed(X:AGENT, phone_usage(T:TYPE))
observed(X:AGENT, social_activity(T:TYPE))

Ontology for belief base: Basic belief (generated belief after several observations on pill intake, alcohol level reading, social activities, phone usage, and blood volume pressure reading)

belief(X:AGENT, pill_intake(F:FREQ_LEVEL))

belief(:X:AGENT, alcohol level(L:LEVEL))

belief(X:X:AGENT, BVP_level (L:LEVEL))

belief(X:AGENT, phone_usage(T:TYPE))

belief(X:AGENT, social_activity(T:TYPE))

Derived belief (belief on substance abuse, avoidant coping, neurotic, social support, immunity and assertiveness)

belief(X:AGENT, sub_abuse(L:LEVEL))

belief(X:AGENT, avoidant_coping(L:LEVEL)

belief(X:AGENT, neurotic(L:LEVEL))

belief(X:AGENT, social_support(T:TYPE))
Table 4

Sort used in the models

\begin{tabular}{|r|l|}
\hline Sort & Elements \\
\hline LEVEL & \{low, medium, high\} \\
\hline TYPE & \{positive, negative\} \\
\hline FREQ_LEVEL & \{normal, not_taken, overdose\} \\
\hline TASK & $\begin{array}{l}\text { \{avoid_substance_abuse, } \\
\text { social_activities, relaxation_activities, } \\
\text { coping_skills, } \\
\text { meet_doctor_therapist }\end{array}$ \\
\hline ROLE & $\begin{array}{l}\text { \{patient, friends_family, } \\
\text { doctor_therapist }\end{array}$ \\
\hline AGENT & \{low, medium, high\} \\
\hline CONDITION & \{risk_relapse, anxiety, healthy $\}$ \\
\hline
\end{tabular}

belief(X:AGENT, immunity(L:LEVEL))

belief(X:AGENT, assertiveness(L:LEVEL))

Ontology for analysis model: There are three levels of analysis used; evaluation on coping skills, social withdrawal, and severe risk factors. These distinctive features provide important information to execute a specific action in the support model.

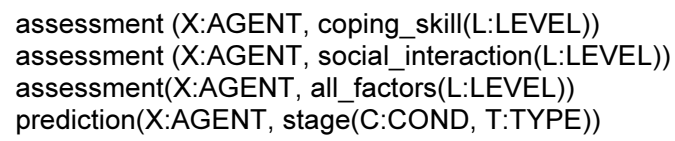

Ontology for support model: Two main actions are used to intervene the risk of relapse namely; notify and advice. The BDI approach regulates action selection process (internal processing) [23]. An action to be taken by an ambient agent is represented using performed as its predicate.

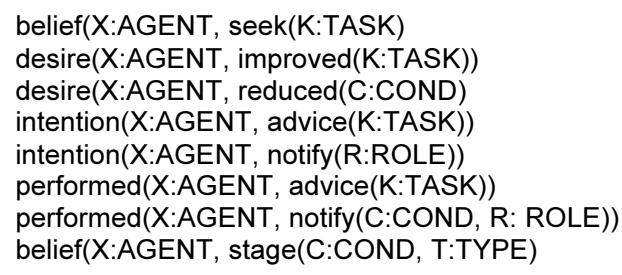

The formalization of some properties makes use of sorts. These sorts are presented in Table 4.

Using this pre-determined ontology, the BeliefDesire-Intention (BDI) approach regulates action selection process (internal processing). To utilize the specification, a forward reasoning method for belief generation is used. It follows the time sequence and causality, to generate new beliefs from previous properties. The ambient agent functionality is described by three actions: belief generation in the belief base, evaluation of risk, and action selection 
for the support. Below a number of related specifications for social withdrawal case are shown.

\section{BB4: Generating basic belief on phone/PDA us- age}

When the ambient agent observes there is no phone/PDA usage,

then the agent will believe that the person is not using phone/PDA to communicate with the others.

observed(agent, phone_usage(negative) $\rightarrow$

belief(agent, phone_usage(negative))

\section{DB5: Derived belief on social support from the phone usage belief}

If the ambient agent believes that there is no phone usage, then the agent will believe there is no social interaction between social support network members.

belief(agent, phone_usage(negative)) $\rightarrow$

belief(agent, social_support(negative))

\section{GE2: Evaluation on social withdrawal condition}

If it is believed that the person is not interacting with any social network support members, and having difficulty to control anger and it is believed that the person is vulnerable for the future onset,

then the agent will conclude that the condition of the person is having social withdrawal.

belief(agent, social_support(negative) $) \wedge$ belief(agent, assertiveness(low)) $\wedge$ belief(agent, immunity(low)) $\rightarrow$ assessment(agent, social_interaction(low))

\section{PCB2: Predicting the risk of relapse from social} withdrawal condition

If the person is having social withdrawal,

then the ambient agent will assess the person as having potential risk of relapse.

assessment(agent, social_interaction(low)) $\rightarrow$

prediction(agent, stage(risk_relapse, positive))

\section{BOR: Belief on relapse}

When the ambient agent predicts that the person is having a risk in relapse,

then the ambient agent will believe that the person is in the risk of relapse.

prediction(agent, stage(risk_relapse, positive)) $\rightarrow$ belief(agent, stage(risk_relapse, positive))

\section{ANR1: Action to notify social support networks}

When the ambient agent believes that the person is in the risk of relapse,

then the ambient agent will notify all friends and family within the social support network.

belief(agent, stage(risk_relapse, positive)) $\rightarrow$ performed(agent, notify(risk_relapse, friends_family))

\section{ANR2: Action to notify the person}

When the ambient agent believes that the person in the risk of relapse, then the ambient agent will notify the person. belief(agent, stage(risk_relapse, positive)) $\rightarrow$ performed(agent, notify(risk_relapse, person))

\section{DSI: Desire to improve social interaction}

If the ambient agent assesses that the person is having social withdrawal,

then the ambient agent will desire to improve person's social interaction by advising the person about suitable social activities.

assessment(agent, social_interaction(low) $) \wedge$ desire(agent, reduced(risk relapse)) $\rightarrow$ desire(agent, improved(social_activities))

\section{ISIA: Intention to advice on social interaction}

When the ambient agent desires to improve the person's social interaction through social activities and the ambient agent believes there is no social interaction between a person and social support network members, then the ambient agent will have an intention to advice the person on suitable social activities.

desire(agent, improved(social_activities)) $\wedge$ belief(agent, social_support(negative)) $\rightarrow$

intention(agent, advice(social_activities))

\section{ASIA: Action to advice on social interaction ac- tivities}

When the ambient agent intends to advice the person regarding to social activities to the person, then the ambient agent will advice the person about those social activities. intention(agent, advice(social_activities)) $\rightarrow$ performed(agent, advice(social_activities))

\section{Simulation results for the integrative agent model}

For the integrated model, a temporal specification language called LEADSTO and its supporting software environment has been used. LEADSTO enables one to model direct temporal relationship between two state properties (dynamic properties). Consider the format of $\alpha \rightarrow \rightarrow_{e, f, g, h} \beta$, where $\alpha$ and $\beta$ are state properties in form of a conjunction of atoms (conjunction of literals) or negations of atoms, and e,f,g,h represents non-negative real numbers. This format can be interpreted as follows:

If state $\alpha$ holds for a certain time interval with duration $g$, after some delay (between $e$ and $f$ ), state property $\beta$ will hold a certain time interval of length $h$.

Here, atomic state properties can have a qualitative, logical format to represent certain observed conditions. In addition, this representation also holds a temporal trace $\gamma$, denoted by 


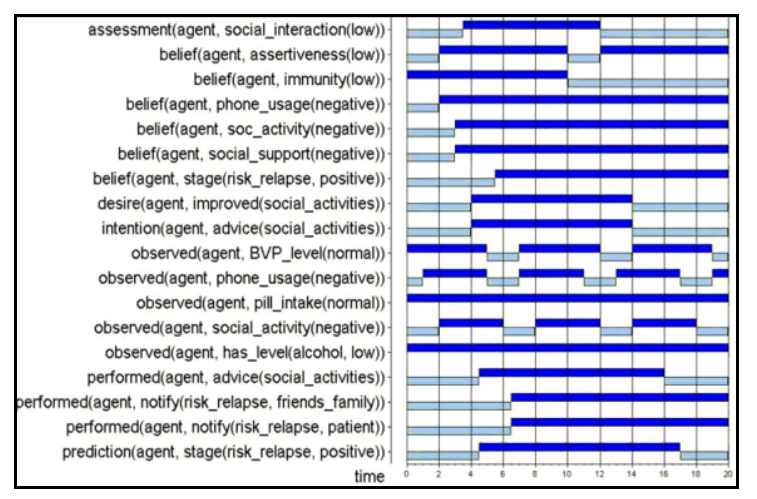

Fig. 10. Simulation trace in social withdrawal.

$$
\begin{aligned}
& \gamma \mid=\alpha \rightarrow e, f, g, h, \text { if } \\
& \forall \mathrm{t} 1[\forall \mathrm{t} 1[\mathrm{t} 1-\mathrm{g} \leq \mathrm{t}<\mathrm{t} 1 \Rightarrow \alpha \text { holds in } \gamma \text { at time } \mathrm{t}] \\
& \Rightarrow \exists \mathrm{d}[\mathrm{e} \leq \mathrm{d} \leq \mathrm{f} \& \\
& \left.\forall \mathrm{t}^{\prime}\left[\mathrm{t} 1+\mathrm{d} \leq \mathrm{t}^{\prime}<\mathrm{t} 1+\mathrm{d}+\mathrm{h}\right] \Rightarrow \beta \text { holds in } \gamma \text { at time } \mathrm{t}^{\prime}\right]
\end{aligned}
$$

For a more detailed discussion of this language, see [7]. Note that LEADSTO is used as a modelling instrument. It also possible to implement the model within any other software environment. Based on the proposed model, using the specified temporal rules to determine the stage of person, several simulations have been performed. For this article, three examples of simulation runs were chosen. In Figs 10-12, timeline is shown on the horizontal axis, the state properties are on the vertical axis and a dark box indicates that a state property is true.

\section{Simulation \# 1: Deficiencies in social interaction}

This condition occurs when the ambient agent observes no activities in social interaction, low in assertiveness, and highly vulnerable towards future onset. The person is highly advised to engage social interaction with others. Having this in motion, social support network members will be informed by an ambient agent (see Fig. 10).

\section{Simulation \#2: Deficiencies in coping skills}

In this simulation, the ambient agent observes several risks, such as: a high blood volume pressure, high alcohol level, and overdose pill intake. Based on this, the agent assesses that the person is facing a risk of relapse, subject to coping skills problem. Therefore, the agent desires to give advice to improve coping skills, specifically to reduce anxiety and later to eliminate substance abuse are translated into intentions. Prior to this, the beliefs about the conditions must hold true. Figure 11 depicts the simulation trace of this condition.

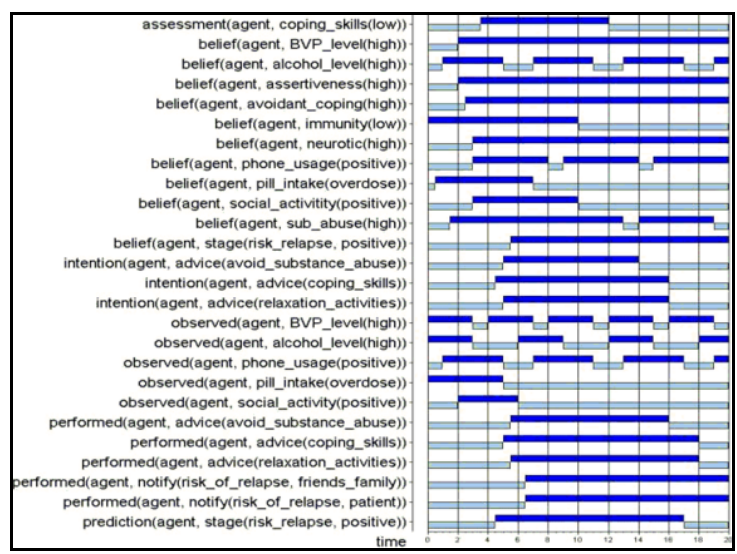

Fig. 11. Simulation trace in coping skills deficiencies.

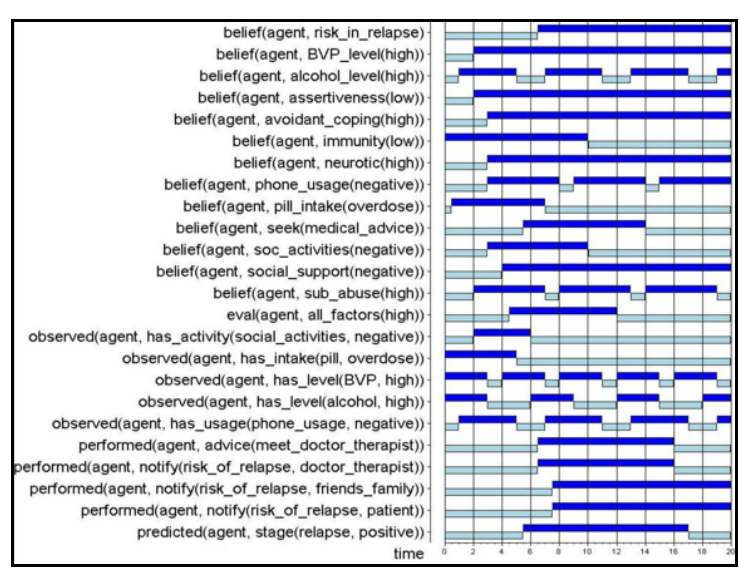

Fig. 12. Simulation trace for all severe risk factors.

\section{Simulation \# 3: Severe risk factors}

The severe risk factors occur when all observed risk factor features show a positive contribution towards the future onset. Normally, seeking medical advice is the only best option. When an ambient agent evaluates a person is having all severe risk factors, the doctor or the therapist will be notified. The person will receive a notification to seek for medical advice. The result of this condition is shown in Fig. 12.

\section{Verification of the integrative agent model}

This section deals with the verification of relevant dynamic properties of the cases considered in the ambient agent model. It is important to verify whether the model produces results that are coherent with the literature and appropriate to help the patient. Several properties have been identified from related 
work in relapse management. The Temporal Trace Langue (TTL) is used to perform an automated verification of specified properties against generated traces. This language allows formal specification and analysis of dynamic properties; it is either a qualitative or a quantitative representation [7].

TTL is designed on atoms, to represent the states, traces, and time properties. This relationship can be presented as a $\operatorname{state}(\gamma, t$, output $(R)) \mid=p$, means that state property $p$ is true at the output of role $R$ in the state of trace at time point $t$. Based on that concept, dynamic properties can be formulated using a sorted predicate logic approach.

VP1: Advice to avoid substance abuse during the risk of relapse

When a person is believed to have a problem in substance abuse, prolong high neurotic level and vulnerable towards relapse (low in immunity),

then the ambient agent provides advice to avoid substance abuse.

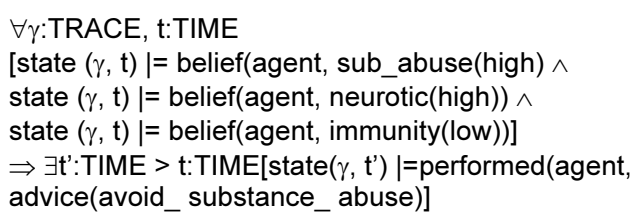

Substance abuse advice needs to be delivered if the persons are showing the risk of relapse, and with the combination of substance abuse problem, vulnerable to the onset, and prolong exposure to the anxiety [31]. It is vital since by prolong exposure towards substance abuse will increase the risk of future onset [21].

VP2: Warn for medical help if all risk conditions have been observed

When the doctor or therapist has been informed, the peron has already had all severe risk factors observed.

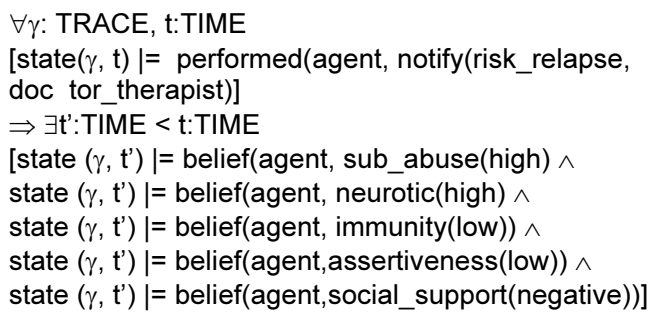

VP3: Social support networks as a buffer for negative life events

When the ambient agent predicts a person is having a risk in relapse,

then the ambient agent sends a notification message to related friends and family within the social support network members.

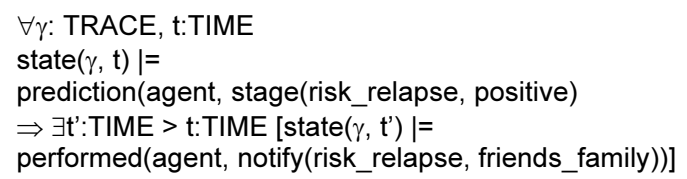

Friends and family within social support networks need to be informed if the person is developing the risk of relapse in future. Ability to have social support is one of the crucial elements to reduce the risk of relapse [11].

VP4: Relaxation training to reduce high comorbidity between anxiety and future onset

If the ambient agent observes a person is having a high reading in blood volume pressure,

then the ambient agent provides advice on relaxation activities

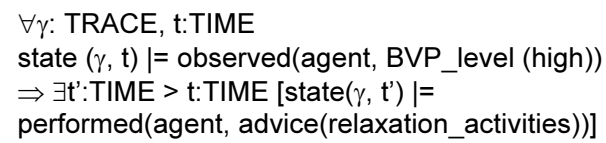

Anxiety can be reduced through a series of relaxation activities $[10,18]$. By reducing the level of anxiety (neurotic), it will deplete the risk of having a relapse.

VP5: Involvement in several social activities to reduce the risk of relapse in the case of social withdrawal

When the ambient agent evaluates a person is having social withdrawal and the ambient agent believes that a person is having no social support,

then the ambient agent will provide advice to engage with suitable social activities.

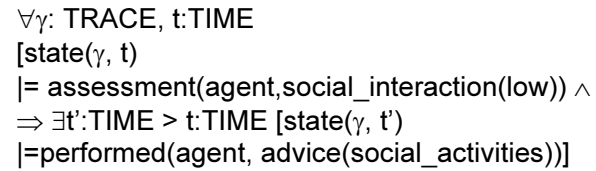

Deficits in social activities increase the chance of relapse. Positive social activities mitigate between stressful life events and onset [11].

\section{Conclusion}

The grand challenge addressed in the research that is reported in this article is to develop an ambient intelligent agent that is capable of monitoring individuals' condition in case of prior depression and providing appropriate suggestions to prevent relapse. In this article, two steps have been taken. Firstly, a dynamical (domain) model was presented that describes the process relapse and recurrence of unipolar depression in humans. This model forms 
the basis for the monitoring function within the ambient agent model; through this domain model it acquires its awareness of the human's state. The proposed domain model is heavily inspired by scientific findings about the relapse and recurrence. The model is able to explain the onset of recurrence and relapse based on personal characteristics and stressor events. It can be used to simulate and analyse different individuals' situations, in relation to their personality and characteristics. A mathematical analysis has been performed to demonstrate the occurrence of equilibrium conditions, fundamentally beneficial to describe convergence and stable states of the model.

Secondly, by embedding knowledge from the domain model into an ambient agent model, an integrative ambient agent model was obtained which is able to reason about the state of the person. Thus, it is capable to predict the risk of relapse based on several observable features and beliefs, mainly using a belief-desire-intention mechanism. The integrative agent model has been specified using a hybrid formal modelling approach, which enables both qualitative and quantitative specification. Within this integrative ambient agent model, some subcomponents are used to provide basic understanding based on monitoring the person's conditions, evaluating the risk, and to decide on actions to sustain the persons' wellbeing. The integration takes place by encapsulating the domain model in these subcomponents. A set of formal temporal properties were specified to allow intelligent reasoning to take place. From this formally specified ambient agent model, several simulation runs were executed using the LEADSTO software environment.

The simulation results have been verified based on several properties using Temporal Trace Language (TTL) environment. The presented model provides a basic design on how an ambient model can be used to monitor person in a risk of relapse and recurrence in unipolar depression. It was shown that the ambient agent model through simulation indeed is capable to provide a support for persons.

Apart from a more thorough evaluation of the proposed models, there are several directions for future research based on the work presented in this article. First, it is beneficial to investigate how interactions and sensing properties can be further developed and enriched to achieve a more fluid embedding into an ambient monitoring system. Second, inclusion of cognitions (for example, the negative cognitive triads) will allow a better understanding of the precursors of relapse in unipolar depression, as the currently presented model only uses some observable behaviours. Special attention could be given to the linkage of cognitive aspects with the existing concepts in the model, such as immunity and neuroticism.

Finally, future work could focus on generalizing the proposed model to a generic model for risk assessment and support in other domains.

\section{References}

[1] G. Andersson, J. Bergstrom, F. Hollandare, P. Carlbring, V. Kaldo and L. Ekselius. Internet-based self-help for depression: randomized controlled trial. The British J. of Psy. 187, (2005). 456-461.

[2] A.A. Aziz, M.C.A. Klein, and J. Treur, An agent model of temporal dynamics in relapse and recurrence in depression. In: Ali, M., Chen, S.M., Chien, B.C., Hong, T.P. (eds.), IEA-AIE 2009. LNAI, Springer Verlag, 2009. pp. 36-45.

[3] A.A. Aziz, M.C.A. Klein, and J. Treur, An ambient intelligent agent model for relapse and recurrence monitoring in unipolar depression. In: Combi, C., Shahar, Y., AbuHanna, A. (eds.), Proceedings of the 12th Conference on Artificial Intelligence in Medicine, AIME'09, LNAI, Springer Verlag, 2009, pp. 186-190.

[4] A.A. Aziz, M.C.A. Klein, and J. Treur, Modelling an ambient agent to support depression relapse prevention. In: Proceedings of the Third International Workshop on Human Aspects in Ambient Intelligence, HAI'09. IEEE Computer Society Press, 2009, pp. 335-340.

[5] G. Belsher, and C.G. Costello, Relapse after recovery from unipolar depression: A Critical Review. Psychological Bulletin. 104, (1988). 84-96.

[6] T. Bickmore, A. Gruber, and R. Picard, Establishing the computer-patient working alliance in automated health behaviour change interventions. Patient Education and Counselling 59 (1), (2005), 21-30.

[7] T. Bosse, C.M. Jonker, L. van der Meij, and J. Treur, A language and environment for analysis of dynamics by simulation. International Journal of Artificial Intelligence Tools, vol. 16, (2007), 435-464.

[8] F. Both, M. Hoogendoorn, M.C.A. Klein, and J. Treur, Design and analysis of an ambient intelligent system supporting depression therapy. In: Luis Azevedo and Ana Rita Londral (Eds), Proceedings of the Second International Conference on Health Informatics, HEALTHINF'09. INSTICC Press, 2009, pp. 142-148.

[9] F. Both, M. Hoogendoorn, M.C.A. Klein, and J. Treur, Formalizing dynamics of mood and depression, In: M. Ghallab, C.D. Spyropoulos, N. Fakotakis and N. Avouris (Eds.), Proc. of the 18th European Conf. on Artificial Intelligence, ECAI'08. IOS Press, 2008, pp. 266-270.

[10] E.I. Brilman, and J. Ormel, Life events, difficulties and onset of depressive episodes in later life, Psychological Medicine 31, (2001). 859-869.

[11] S. Cohen, and T.A. Wills, Stress, social support, and the Buffering Hypothesis, Psychological Bulletin 98 (1985), 310-357.

[12] D.J. Cook, Multi-agent smart environments. Journal of Ambient Intelligence and Smart Environments. Vol. 1 (2009), 51-55. 
[13] A.K. Dey, Modelling and intelligibility in ambient environments. Journal of Ambient Intelligence and Smart Environments. Vol. 1 (2009), 57-62.

[14] S.H. Fairclough, Fundamentals of physiological computing, Interacting with computers 21 (2009), no. 1-2, 133145 .

[15] G.H.E. Gendolla, On the impact of mood on behaviour: An integrative theory and a review, Review of general psychology. Vol. 4 (2000), no. 4, 378-408.

[16] A.P. Glascock, and D.M. Kutzik, The impact of behavioural monitoring technology on the provision of health care in the home. Journal of Universal Computer Science 12(1), (2006), 59-79.

[17] D.J. Green, Real-time compliance management using a wireless real-time pill bottle - a report on the pilot study of SIMPILL. In: Proc. of the International Conference for eHealth, Telemedicine and Health'05, 2005.

[18] K.C. Gunthert, L.H. Cohen, and S. Armeli, The role of neuroticism in daily stress and coping, Journal of Personality and Social Psychology 77, (1999), 1087-1100.

[19] R.E. Ingram, and D.D. Luxton, Vulnerability-Stress models, In J. Abel (Ed.). Development of Psychopathology: Stress-Vulnerability Perspectives, New York, (2005), 3246.

[20] M.B. Keller, The long-term treatment of major depression. J. Clin. Psychiatry (1999). 41-45.

[21] L.V. Kessing, M.G. Hansen, P.K. Andersen, and J. Angst, The predictive effect of episodes on the risk of recurrence in depressive and bipolar disorders - a life-long perspective. Acta Psychiatrica Scandinavica 109, (2004), 339-344.

[22] R.C. Kessler, The effects of stressful life events on depression, Annual Review of Psychology 48, (1997), 191-214.

[23] D. Kinny, M. Georgeff, and A. Rao, A methodology and modelling technique for systems of BDI agents, Proceedings of the 7th European Workshop on Modelling Autonomous Agents in a Multi-Agent World, LNAI Volume 1038, (1999). pp. 56-71.

[24] P.M. Lewinsohn, H. Hoberman, L. Teri, and M. Hautzinger, An integrative theory of depression, In S. Reiss and R.R. Bootzin (Eds.), Theoretical Issues in Behaviour Therapy, (1985), 331-352.
[25] S.M. Monroe, and K.L. Harkness, Life stress, the kindling hypothesis and the recurrence of depression: consideration from a life stress perspective, Psy. Review 112:2, (2005), 417-445.

[26] A.A. Neirenberg, T.J. Petersen, and J.E. Alpert, Prevention of relapse and recurrence in depression: the role of longterm pharmacotherapy and psychotherapy. J. Clinical Psychiatry 64(15), 2003, pp. 13-17.

[27] S.A. Neumann and S.R. Waldsein, Similar patterns of cardio-vascular response during emotional activation as a function of affective valence and arousal and gender, Journal of psychosomatic research 50 (2001), no. 5, 245-253.

[28] J. Ormel, and T. Wohlfarth, How neuroticism, long term difficulties, and life situation change influence psychological distress: a longitudinal model. Journal of Personality and Social Psychology 60(5), (1991), 744-755.

[29] R.W. Picard, and K.K. Liu, Relative subjective count and assessment of interruptive technologies applied to mobile monitoring of stress, International Journal of HumanComputer Studies. Vol. 65(4), (2007), 361-375.

[30] R.W. Picard, E. Vyzas, and J. Healey, Towards machine emotional intelligence: analysis of affective physiological state, IEEE Transactions on Pattern Analysis and Machine Intelligence. Vol. 23 (10), (2001), 1175-1191.

[31] G. Shahar, J. Joiner, D.C. Zuroff, and S.J. Blatt, Personality, interpersonal behaviour, and depression: co-existence of stress-specific moderating and mediating effects, Personality and Individual Differences (36), (2004), 15831596.

[32] J. Treur, On Human Aspects in Ambient Intelligence. In: Proceedings of the First International Workshop on Human Aspects in Ambient Intelligence. Published in: M. Mühlhäuser, A. Ferscha, and E. Aitenbichler (eds.), Constructing Ambient Intelligence: AmI-07 Workshops Proceedings. Communications in Computer and Information Science (CCIS), Springer Verlag, 2008, pp. 262-267.

[33] J. Zhai, and A.B. Barreto, Instrumentation for automatic monitoring of affective state in human-computer interaction, In 18th International Florida Artificial Intelligence Research Society Conference, 2005, pp. 207-212. 